\title{
Níveis de Concordância com Regras Descritivas sobre Comportamentos Alimentares entre Veg(etari)anos
}

\author{
Rodrigo Clementino de Carvalho ${ }^{1}$ \\ Júnnia Maria Moreira ${ }^{1}$ \\ ${ }^{1}$ Universidade Federal do Vale do São Francisco, Petrolina, Pernambuco, Brasil
}

\begin{abstract}
Resumo
O vegetarianismo é uma prática que exclui carnes e pode evitar outros produtos de origem animal. Esta última modalidade está muito associada ao veganismo, uma filosofia que defende a libertação animal. Aderir a tais condições implica no seguimento de algumas normas que norteiam comportamentos alimentares e interações sociais. Partindo desse pressuposto, objetivou-se avaliar o nível de concordância, entre vegetarianos e veganos, quanto a regras que descrevem comportamentos alimentares. A pesquisa contou com 398 participantes, sendo 198 não estritos e 200 estritos, que responderam a um questionário on-line autoaplicável. Os dados, analisados via teste $t$, apontaram maiores níveis de concordância com comportamentos mais restritos entre vegetarianos estritos e veganos os quais também relataram maior embasamento em valores. Este estudo, que trouxe uma ótica de análise para os comportamentos alimentares em termos de localização de reforços, pode contribuir com novas práticas em saúde direcionadas às pessoas vegetarianas e veganas.

Palavras-chave: dieta vegetariana, dieta vegana, comportamento alimentar, comportamento governado por regras
\end{abstract}

Levels of Agreement With Descriptive Rules On Food Behavior Between Vegans and Vegetarians

\begin{abstract}
Vegetarianism is a practice that excludes meat and can avoid other products of animal origin. This latter modality is closely associated with veganism, a philosophy that advocates animal liberation. Adherence to these conditions implies following some norms that guide eating behaviors and social interactions. Based on this assumption, we aimed to evaluate the agreement level, between vegetarians and vegans, regarding rules that describe food behaviors. The survey included 398 participants, 198 nonstrict and 200 strict vegetarians, who answered a self-administered online questionnaire. The data, analyzed by t-test, showed higher agreement level with more restricted behaviors between strict vegetarians and vegans, who also reported a higher base on values. This study, which provided an analysis perspective on eating behaviors in terms of reinforcement location, may contribute to new health practices aimed at vegetarians and vegans.

Keywords: vegetarian diet; vegan; food behavior; rule-governed behavior
\end{abstract}

\section{Niveles de Acuerdo con Reglas Descriptivas Sobre Comportamientos Alimentarios Entre Veg(etari)anos}

\section{Resumen}

El vegetarianismo es una práctica que excluye carnes y también puede evitar otros productos de origen animal de la alimentación. Esta última modalidad está muy asociada al veganismo, una filosofía que defiende la liberación animal. Adherirse a tales condiciones implica el seguimiento de algunas normas que guían los comportamientos alimentarios e interacciones sociales. A partir de esta suposición, el objetivo fue evaluar el nivel de acuerdo, entre vegetarianos y veganos, en cuanto a las reglas que describen conductas alimentarias. La encuesta contó con 398 participantes, siendo 198 no estrictos y 200 estrictos, que respondieron a un cuestionario online autoaplicable. Los datos, analizados vía test T, señalaron mayores niveles de acuerdo con comportamientos más restringidos entre vegetarianos estrictos y veganos, los cuales también señalaron una mayor base en los valores. Este estudio, que trae una óptica de análisis para los comportamientos alimentarios en términos de localización de refuerzos, puede contribuir con nuevas prácticas en salud dirigidas a personas vegetarianas y veganas.

Palabras clave: dieta vegetariana; dieta vegana; conducta alimentaria; conducta gobernada por reglas

O vegetarianismo é concebido, de forma ampla, como uma prática dietética que exclui alimentos cárneos (partes de corpos de animais) e que pode ou não evitar outros produtos de origem animal (Sociedade Vegetariana Brasileira, 2014). Em seu modo mais estrito, o vegetarianismo costuma fazer parte do veganismo, uma filosofia que recusa, na medida do possível e do praticável, qualquer produto, serviço, atividade ou evento que envolva o sofrimento animal (Ferrigno, 2012; The Vegan
Society, 2014). O veganismo, então, engloba e extrapola o vegetarianismo, que alude ao nível alimentar.

Atualmente, o número de vegetarianos, nos quais se incluem os veganos, difere dentre os países pesquisados (Esteves, 2017; Ruby, 2012). No Brasil, 14\% da população brasileira acima dos 16 anos se diz vegetariana, tendo esse número crescido em relação ao levantamento de 2012 (Ibope, 2018). Apesar da porcentagem parecer pequena, ela representa quase 30 milhões 
de adeptos ao vegetarianismo no país, segundo o último levantamento, o que torna o público vegetariano uma minoria expressiva (Ruby, 2012).

Apesar de algumas limitações, a literatura apresenta algumas propostas de avaliação dos regimes vegetarianos (Beardsworth \& Keil, 1992; Rosenfeld \& Burrow, 2017a; Rosenfeld \& Burrow, 2018). Com base nas características dietéticas, o vegetarianismo se classifica em: ovolactovegetarianismo, no qual ovos e laticínios são consumidos; lactovegetarianismo, quando os laticínios participam da alimentação; ovovegetarianismo, no qual se mantém o consumo de ovos; e vegetarianismo estrito, quando se exclui todos os produtos de origem animal (Sociedade Vegetariana Brasileira, 2014)

Quanto aos objetivos que embasam uma dieta vegetariana, podem ser elencados três principais: os pró-sociais, quando se pretende beneficiar algo para além do próprio sujeito (exemplo: animais e meio ambiente); os objetivos pessoais, quando o sujeito vegetariano privilegia o benefício próprio; e objetivos morais, quando o vegetarianismo se baseia em noções de "certo" ou "errado". Essas orientações podem ocorrer ou não em conjunto (Rosenfeld \& Burrow, 2017a). O vegetarianismo poderia se basear ainda em aversões, quando é fruto de experiências negativas com carnes e em restrições, quando é resultado da falta de acesso a produtos cárneos (Rosenfeld \& Burrow, 2017b).

Outra classificação se baseia nas razões para se tornar vegetariano. Em sua revisão bibliográfica, Ruby (2012) identificou que a dimensão ética, referente à causa animal, é o principal argumento que conduz a uma vida vegetariana. $\mathrm{Na}$ sequência, são elencados: saúde pessoal, preocupação ambiental, pureza espiritual e repulsa pelas propriedades da carne, como sabor. As motivações para se manter vegetariano também englobam essas instâncias, que podem ser acrescidas, excluídas ou alteradas com o tempo, configurando o vegetarianismo de razões mistas (Beardsworth \& Keil, 1992; Rothgerber, 2014). Tais motivações também estariam relacionadas ao grau de restrição do estilo vegetariano adotado (Hoffman, Stallings, Bessinger, \& Brooks, 2013; Rosenfeld, 2018; Ruby, 2012).

Esses motivos são citados em alguns estudos que associam o consumo de carne a riscos para a saúde humana (Wolk, 2017) e a prejuízos ambientais (Aleksandrowicz, Green, Joy, Smith, \& Haines, 2016). Outros malefícios envolveriam a poluição espiritual, segundo algumas vertentes religiosas (Ruby, Heine, Kamble, Cheng, \& Waddar, 2013), e a eliciação de nojo frente às propriedades físicas das carnes (Arora,
Bradford, Arora, \& Gavino, 2017; Rothgerber, 2014). Por outro lado, os benefícios de ser vegetariano se situariam na possibilidade dessa alimentação ser segura e saudável, desde que seguida de forma adequada, o que é esperado de qualquer prática dietética (Corrin \& Papadopoulos, 2017; Melina, Craig, \& Levin, 2016; Zuromski et. al., 2015).

A permanência da condição vegetariana envolveria ainda aspectos pessoais, como bem-estar e controle de peso corporal; suporte social de familiares, amigos e grupos de incentivo ao vegetarianismo; e disponibilidade de itens de consumo vegetarianos no mercado (Jabs, Devine, \& Sobal, 1998). Tais dados, oriundos de uma pesquisa internacional, podem não refletir o cenário brasileiro, que apresenta escassos estudos em Psicologia sobre o vegetarianismo (Esteves, 2017; Lima, 2018).

As práticas vegetarianas, envolvendo comportamentos, podem ser compreendidas sob a ótica da Psicologia Analítico-Comportamental que enfoca o comportamento humano e suas relações com o ambiente. Todo comportamento, segundo Skinner (1981/2007), resulta do conjunto de processos nos níveis filogenético, ontogenético e cultural. Um alimento com valor evolutivo, a exemplo da carne, pode não ser reforçador para um certo organismo, que terá seus comportamentos alimentares sujeitos à influência da sua história de vida e do cenário cultural (Ruby et al., 2013).

Entende-se por comportamentos alimentares as ações que envolvem o ato de se alimentar, permeadas por fatores psicológicos, socioculturais, dentre outros, para além dos fisiológicos e das características dos alimentos. A alimentação não mais se restringe ao suprimento de necessidades nutricionais, tendo outras funções ligadas a relações sociais. Tais circunstâncias podem influenciar o comportamento alimentar, público ou privado, e afetar comportamentos futuros que podem ficar sob o controle de regras (Albuquerque, 2001; Reis, Teixeira, \& Paracampo, 2005).

Jabs, Sobal e Devine (2000) identificaram regras associadas ao vegetarianismo que, para além de uma prática dietética, constituiria e manifestaria a noção de identidade (Lima, 2018; Rosenfeld \& Burrow, 2018). Nesse sentido, adotar um estilo vegetariano pressuporia o seguimento de certas normas que, por sua vez, influenciariam as práticas alimentares e relações interpessoais de um indivíduo (Jabs, Sobal, \& Devine, 2000). A violação dessas regras poderia eliciar sensações de ansiedade, culpa e raiva (Hamilton, 2006). Mediante tais 
considerações, este estudo foi proposto para verificar o nível de concordância, entre vegetarianos e veganos, quanto a regras que descrevem comportamentos alimentares.

\section{Método}

\section{Participantes}

A pesquisa foi realizada com 398 participantes, sendo 198 vegetarianos não estritos (VNE) e 200 vegetarianos estritos e/ou veganos (VE), que apresentaram médias de idade de 28,5 anos $(D P=10,1)$ e 31,5 anos $(D P=11,1)$, respectivamente.

\section{Instrumentos}

Foi utilizado como instrumento para a coleta de dados um questionário autoaplicável on-line, criado pelos pesquisadores com o auxílio da plataforma Google Forms. $\mathrm{Na}$ primeira página do questionário havia o Termo de Consentimento Livre e Esclarecido (TCLE), seguido por itens referentes à frequência de consumo de produtos de origem animal. Caso houvesse registro de consumo frequente ou ocasional de algum tipo de carne e/ou subprodutos (vermelha, aves, gordura animal, embutidos, peixes e frutos do mar) o questionário era encerrado, uma vez que a definição de vegetarianismo pressupõe abstenção completa de produtos derivados da estrutura corporal de animais.

Dos 496 voluntários que concordaram com o TCLE, prosseguiram os 403 que revelaram não comer nenhum tipo de carne, consumindo ou não outros alimentos de origem animal (ovos, laticínios, mel, gelatina, cochonilha e/ou outro corante de origem animal e caviar). Em seguida, foi questionado sobre o tipo de dieta vegetariana seguida: não estrita, quando algum outro produto de origem animal que não fosse cárneo ainda era consumido, ou estrita, caso rejeitasse todos os produtos de origem animal. Essa informação direcionou cada participante ao questionário referente ao seu estilo alimentar. Foram então solicitados dados sociodemográficos dos participantes e informações referentes às suas práticas alimentares, como há quanto tempo as adotam e suas razões. As perguntas, em sua maioria, eram de múltipla escolha, porém algumas permitiam comentários. Cinco questionários foram excluídos por erros de preenchimento, o que ocasionou em um total de 398 participantes.

Posteriormente, o participante deveria considerar itens sobre comportamentos alimentares e situações sociais relacionadas, construídos com base na literatura sobre o tema (Rosenfeld \& Burrow, 2017a, 2017b; Ruby, 2012) e nas experiências de vida do primeiro autor, que é vegetariano. Para cada item o participante deveria indicar seu nível de concordância em uma escala Likert de cinco pontos, variando de "discordo totalmente" a "concordo totalmente", sendo maior concordância indicativa de maior restrição comportamental. Quanto às afirmativas invertidas $(2,3,6,7,8,9,10,12,13,14,15,19,21$, $27,32,35,36)$, a escala de resposta foi alterada para se manter a correspondência entre maiores concordâncias com as afirmativas e comportamentos mais restritos. Portanto, maiores médias indicam maior discordância nos itens invertidos. Após responder a todos os itens, o questionário era encerrado e a participação finalizada.

\section{Procedimento}

Os participantes foram acessados por meio de redes sociais, especialmente em grupos do Facebook que reúnem praticantes da alimentação vegetariana, associada ou não ao veganismo e também via Whatsapp. Foram feitas divulgações contendo os objetivos da pesquisa, juntamente com o link para o questionário on-line.

\section{Análise de Dados}

Os dados sociodemográficos e referentes aos hábitos alimentares foram analisados quanto à frequência (porcentagem e média). Diferenças em termos da concordância com os itens entre os grupos VNE e VE foram analisadas por meio do teste $t$, utilizando-se o software SPSS 22.

Quanto aos aspectos éticos, a pesquisa foi realizada de acordo com os critérios da Resolução 510/2016 (Brasil, 2016). Adaptou-se o TCLE para que o participante tivesse acesso ao instrumento somente após declarar ciência dos termos da pesquisa e autorizar a sua participação no estudo. Foi disponibilizado também um e-mail, para que os participantes pudessem requerer qualquer reparação de danos. Não houve coleta e retenção de dados que identificassem os participantes, o que garantiu o anonimato.

\section{Resultados e Discussão}

Como mostra a Tabela 1, a amostra foi composta em sua maioria por mulheres (79,3\% VNE, $82 \% \mathrm{VE})$, que tiveram acesso ao ensino superior $(89,9 \% \mathrm{VNE}$, $88,5 \% \mathrm{VE})$, com renda pessoal de até três salários míni$\operatorname{mos}(63,6 \% \mathrm{VNE}, 52 \% \mathrm{VE})$ e sem religião $(67,7 \%$ VNE, 69\% VE). Dentre os que declararam alguma religião, destacou-se o espiritismo ou kardecismo 
(9,6\% VNE, 13\% VE). A maioria dos participantes segue seu atual estilo alimentar há menos de três anos (58,1\% VNE, 64,5\% VE). No grupo VNE, a alimentação ovolactovegetariana foi a mais comum $(77,3 \%)$, tendo a maioria desse grupo manifestado interesse em ser vegetariano estrito ou vegano no futuro $(67,2 \%)$. A maior parte do grupo VNE $(87,9 \%)$ se considerou vegetariana e a maioria do grupo $\mathrm{VE}$, majoritariamente, intitulou-se vegana $(83 \%)$. Os demais participantes preferiram termos como ovolactovegetariano(a) e vegetariano(a) estrito(a), além de outros, como simpatizante, protovegetariano(a), em transição ou adepto de uma alimentação plant-based. Quanto à transição para o atual regime alimentar, foi mais comum o modo abrupto no grupo VNE (56,1\%) e a transição gradativa no grupo VE $(55,5 \%)$.

Esses dados estão alinhados com a literatura que descreve os vegetarianos como majoritariamente jovens, de sexo feminino e com maiores níveis de escolaridade (Pfeiler \& Egloff, 2018; Rosenfeld, 2018; Ruby, 2012). A renda pessoal de até três salários mínimos, prevalente entre ambos os grupos, converge com o dado sobre o vegetarianismo estar se popularizando entre pessoas de baixa renda (Allès et al., 2017). A ausência de religião na maior parte dos vegetarianos e veganos revela a independência desses estilos de vida quanto a correntes religiosas, o que é característico da contemporaneidade especialmente em sociedades ocidentais (Hamilton, 2006).

A Figura 1 retrata os motivos mais citados para o início e manutenção de uma alimentação vegetariana. Os resultados corroboram com o levantamento bibliográfico de Ruby (2012), que traz a instância ética como principal motivo que endossa hábitos vegetarianos, a exemplo da causa animal, seguida pelas questões relativas à preservação ambiental, saúde pessoal, aversão ao sabor e a outras características dos produtos de origem animal e aspectos religiosos ou espirituais. Alguns participantes da amostra em ambos os grupos relataram eventos específicos que influenciaram seu comportamento alimentar, como a adoção de um animal de estimação e contato com abate ou documentário sobre a produção de alimentos de origem animal. Outros aspectos, como influência de familiares e amigos, estética e economia financeira foram pouco apontados entre os participantes, sendo mais comuns menções a aspectos éticos, ecológicos, políticos, socioeconômicos, filosóficos e espirituais, remetendo a objetivos pró-sociais e morais (Rosenfeld \& Burrow, 2017a).
Quase todos os motivos foram mais citados na manutenção da condição vegetariana do que em seu início. A quantidade média de motivos, por exemplo, subiu de 3,7 $(D P=1,8)$ para 4,6 $(D P=2,2)$ no grupo VNE e de 3,2 (DP = 1,8) para 4,2 $(D P=2,3)$ no grupo VE, apontando que vegetarianos acrescentam ao longo do tempo novos motivos aos originários (Beardsworth \& Keil, 1992; Hamilton, 2006). Esse fato poderia ser influenciado por novas experiências após ingressar no vegetarianismo e no veganismo, solidificando a permanência no estilo de vida adotado. Os motivos que mais cresceram foram saúde pessoal, preferência pela culinária vegetariana e repulsa pelas propriedades dos alimentos de origem animal, que tendem a somar com as questões éticas e configurar um vegetarianismo de razões mistas (Beardsworth \& Keil, 1992; Rothgerber, 2014).

Outro fator de manutenção para um estilo de vida vegetariano trazido pela literatura é o apoio familiar. A maioria dos participantes do presente estudo se sente apoiada por pessoas próximas $(53 \% \mathrm{VNE}, 61,5 \% \mathrm{VE})$, porém não foi possível verificar se isso ocorre desde o início da mudança alimentar. $\mathrm{O}$ apoio familiar, ao mesmo tempo em que ajuda a manter uma condição vegetariana ou vegana, pode interferir nesse processo, sendo comum uma ambivalência quanto a acolher ou reprovar a mudança alimentar no seio familiar (Jabs et al., 1998; Lima, 2018).

Os níveis de concordância com os itens, médias e desvios padrão dos grupos em geral e por item, diferenças estatísticas entre os grupos e tamanhos do efeito são apresentados na Tabela 2. O tamanho do efeito foi avaliado por meio do $d$ de Cohen. Efeitos maiores ou iguais a 0,70 foram considerados grandes, entre $0,30 \mathrm{e}$ 0,69 médios e menores que 0,29 pequenos.

O grupo VE apresentou média geral de concordância maior comparado ao grupo $\operatorname{VNE}(d=0,87)$, o que sugere comportamentos mais restritivos no primeiro grupo. Essa restrição, porém, pode não implicar em insensibilidade às contingências, uma vez que vegetarianos, incluindo veganos, seriam mais abertos a novas experiências quando comparados a onívoros (Forestell \& Nezlek, 2018; Pfeiler \& Egloff, 2018). Essa restrição pode significar maior sensibilidade às consequências de longo prazo e maiores índices de autocontrole (Reis, Teixeira, \& Paracampo, 2005). Em outras palavras, o grupo VE seria menos suscetível a consequências imediatas, como o consumo de carne ou outro produto de origem animal, em prol de reforços futuros e de maior magnitude como a redução de impactos ambientais 
Tabela 1.

Características dos Participantes (em \%)

\begin{tabular}{|c|c|c|}
\hline & $\begin{array}{c}\text { VNE } \\
(N=198)\end{array}$ & $\begin{array}{c}\text { VE } \\
(N=200)\end{array}$ \\
\hline \multicolumn{3}{|l|}{ Sexo } \\
\hline Masculino & 20,7 & 18 \\
\hline Feminino & 79,3 & 82 \\
\hline \multicolumn{3}{|l|}{ Escolaridade } \\
\hline Médio completo ou incompleto & 10,1 & 11,5 \\
\hline Superior completo ou incompleto & 89,9 & 88,5 \\
\hline \multicolumn{3}{|l|}{ Renda pessoal } \\
\hline Até 3 salários mínimos & 63,6 & 52 \\
\hline Acima de 3 salários mínimos & 36,4 & 48 \\
\hline \multicolumn{3}{|l|}{ Religião } \\
\hline Não tem & 67,7 & 69 \\
\hline Espírita/kardecista & 9,6 & 13 \\
\hline Católica & 8,1 & 3,5 \\
\hline Protestante/evangélico/adventista & 4,5 & 1,5 \\
\hline Cristã (sem especificar) & 3,0 & 2 \\
\hline Outra & 7,1 & 11 \\
\hline \multicolumn{3}{|c|}{ Há quanto tempo você adota seu estilo de vida atual? } \\
\hline Até três anos & 58,1 & 64,5 \\
\hline Acima de três anos & 41,9 & 35,5 \\
\hline \multicolumn{3}{|l|}{ Qual o seu atual estilo alimentar? } \\
\hline Ovolactovegetariano & 77,3 & - \\
\hline Lactovegetariano & 13,1 & - \\
\hline Ovovegetariano & 8,1 & - \\
\hline Vegetariano estrito & - & 100 \\
\hline Outro & 1,5 & - \\
\hline \multicolumn{3}{|c|}{ Você deseja ser vegetariano estrito ou vegano no futuro? } \\
\hline $\operatorname{Sim}$ & 67,2 & - \\
\hline Não sei & 28,3 & - \\
\hline Não & 4,5 & - \\
\hline \multicolumn{3}{|c|}{ Atualmente, você se considera vegetariano (para VNE) / vegano (para VE)? } \\
\hline $\operatorname{Sim}$ & 87,9 & 83 \\
\hline Não & 12,1 & 17 \\
\hline \multicolumn{3}{|c|}{ Como foi a transição para o seu estilo de vida atual? } \\
\hline Abrupta & 56,1 & 44,5 \\
\hline Gradativa & 43,9 & 55,5 \\
\hline
\end{tabular}

e melhores níveis de saúde em médio e longo prazo (Aleksandrowicz et al., 2016), porém essa possibilidade não foi avaliada.
O grupo VE apresentou menor discordância do item 6 (Apesar de vegetariano/vegano, não sinto nojo ou repulsa ao ver carnes), o que denota menor repulsa 


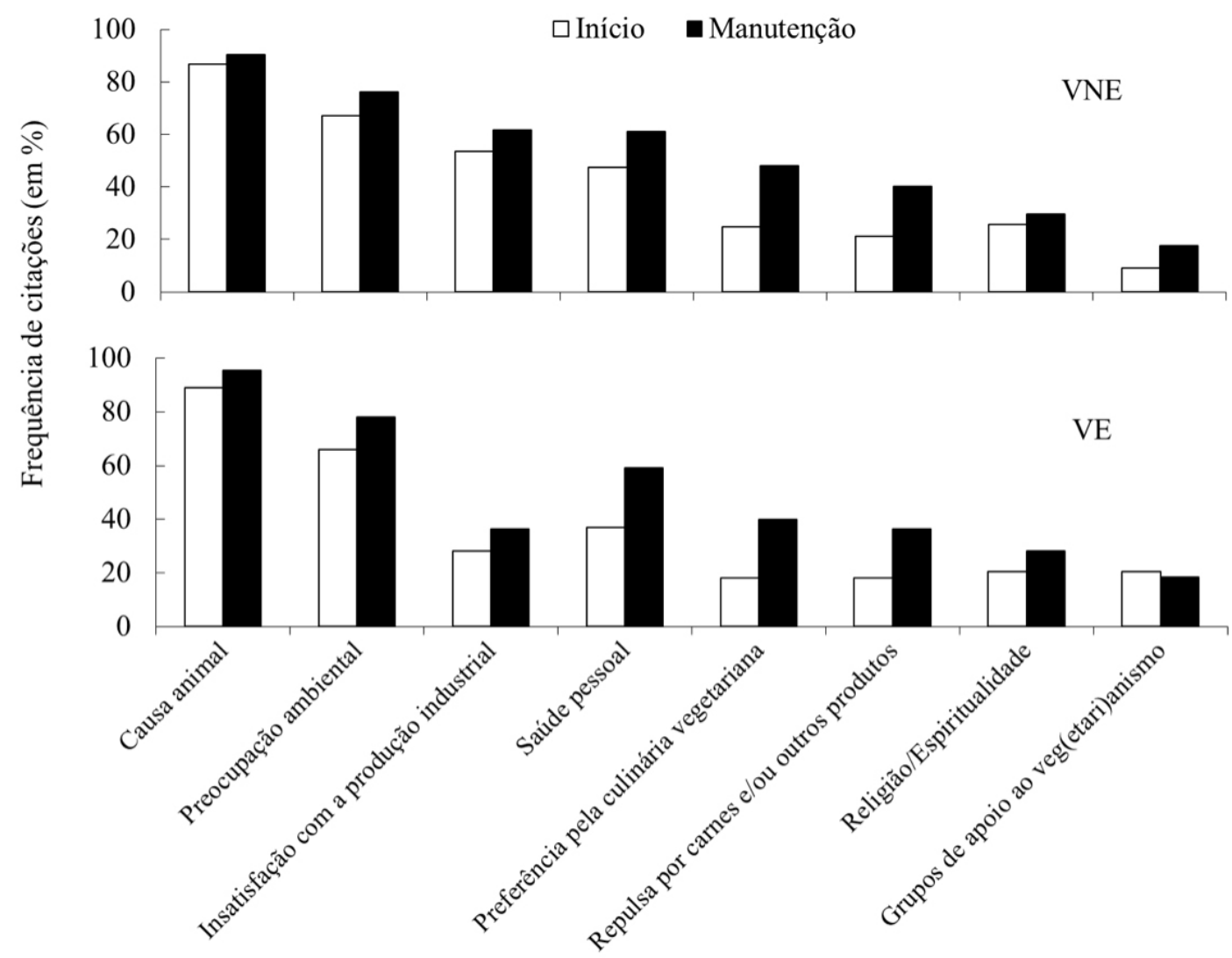

Figura 1. Motivos mais citados para o início e manutenção de uma alimentação vegetariana entre os grupos VNE e VE.

ao ver carne que o grupo VNE. Transições para o vegetarianismo não estrito, que podem ser influenciadas mais por aversão às carnes do que por valores éticos, poderiam estar associados a esse dado. O grupo VE também apresentou maior discordância com o item 15 (Apesar de vegetariano/vegano, o cheiro de carne não me incomoda) revelando maior desconforto quando expostos ao cheiro de carne, o que converge com achados que apontam maior aversão, entre o grupo mais restrito, quanto às propriedades das carnes (Arora et al., 2017; Rosenfeld, 2018; Rothgerber, 2014).

Sentimentos provocados por contato com carne aparecem também na maior concordância no grupo VE com o item 26 (Chego a sentir raiva ou tristeza quando vejo carnes, pois lembro de onde elas vieram) pode ser devido à associação entre o alimento e a morte de animais (Rosenfeld, 2018). De forma similar, o grupo VE concordou mais que o VNE quanto ao item 1 (Certas razões para ser vegetariano/vegano são mais válidas que outras); quanto ao item 24 (Mesmo que um animal estivesse no fim da vida, eu não o comeria); quanto ao item 29 (Pessoas que evitam carnes por questões religiosas/espirituais só serão vegetarianas se pensarem nos animais); e quanto ao item 33 (Quem não come carnes por influência de outras pessoas só será vegetariano se entender as razões desse movimento), porém com efeito baixo $(0,28 ; 0,22 ; 0,25$; e 0,22 , respectivamente). Esses resultados apontam para uma maior priorização de preceitos éticos no veganismo (The Vegan Society, 2014), que não necessariamente são destacados na condição vegetariana (Ruby, 2012; Rosenfeld, 2018), mas tal diferença foi pouco expressiva entre os grupos.

Outra diferença entre os grupos que suporta essa afirmação foi a encontrada quanto ao item 36 (Eu comeria uma certa carne, sabendo que veio de um animal que teve vida digna e abate humanizado), no qual o grupo VE apresentou maior discordância que o grupo VNE, com efeito médio $(0,59)$. Porém, é importante mencionar que esse item foi um dos que apresentaram maiores níveis de discordância em ambos os grupos. Isso remete à ideia do abolicionismo ou libertação 
Tabela 2.

Médias Geral e por Itens para Cada Grupo, com Valores de Comparação de Médias

\begin{tabular}{|c|c|c|c|}
\hline Itens do questionário & $\begin{array}{c}\mathrm{VNE} \\
(\mathrm{N}=198) \\
M(D P)\end{array}$ & $\begin{array}{c}\mathrm{VE} \\
(\mathrm{N}=200) \\
M(D P)\end{array}$ & $t / d$ \\
\hline Média geral para todos os itens & $3,1(0,4)$ & $3,5(0,4)$ & $-8,72 * / 0,87$ \\
\hline $\begin{array}{l}\text { 1. Certas razões para ser vegetariano/vegano são mais válidas que } \\
\text { outras. }\end{array}$ & $2,8(1,4)$ & $3,2(1,5)$ & $-2,81 * / 0,28$ \\
\hline $\begin{array}{l}\text { 2. Qualquer pessoa que não come carnes pode ser chamada de } \\
\text { vegetariana. }^{1}\end{array}$ & $2,6(1,4)$ & $3,5(1,6)$ & $-5,51 * / 0,59$ \\
\hline $\begin{array}{l}\text { 3. Qualquer pessoa que não come produtos de origem animal } \\
\text { pode ser chamada de vegana. }{ }^{1}\end{array}$ & $2,6(1,5)$ & $3,5(1,6)$ & $-6,26 * / 0,62$ \\
\hline $\begin{array}{l}\text { 4. Vegetarianos são mais respeitáveis que os não-vegetarianos, } \\
\text { porque não se alimentam de animais. }\end{array}$ & $1,9(1,4)$ & $2,0(1,1)$ & - \\
\hline $\begin{array}{l}\text { 5. Veganos são mais admiráveis que vegetarianos, pois não } \\
\text { dependem dos animais para se alimentarem. }\end{array}$ & $2,3(1,4)$ & $2,7(1,4)$ & $-2,68 * / 0,27$ \\
\hline $\begin{array}{l}\text { 6. Apesar de ser vegetariano/vegano, não sinto nojo ou repulsa ao } \\
\text { ver carnes. }{ }^{1}\end{array}$ & $2,7(1,5)$ & $2,1(1,3)$ & $4,15^{*} / 0,41$ \\
\hline $\begin{array}{l}\text { 7. Pode ser chamado de vegetariano quem não come carne } \\
\text { vermelha e aves, mas come peixes ou frutos do mar. }{ }^{1}\end{array}$ & $4,8(0,6)$ & $4,9(0,5)$ & - \\
\hline $\begin{array}{l}\text { 8. Quem não come carnes, e mantém o consumo de ovos ou de } \\
\text { laticínios, pode ser chamado de vegetariano. }{ }^{1}\end{array}$ & $2,1(1,3)$ & $3,3(1,6)$ & $-7,67 * / 0,77$ \\
\hline $\begin{array}{l}\text { 9. Um vegetariano que comeu peixe ou fruto do mar errou menos } \\
\text { do que outro que comeu carne vermelha ou de ave. }{ }^{1}\end{array}$ & $4,4(1,0)$ & $4,7(0,8)$ & $-2,59 * / 0,26$ \\
\hline $\begin{array}{l}\text { 10. Um vegano que comeu ovo ou laticínio errou menos do que } \\
\text { outro que consumiu alguma carne. }{ }^{1}\end{array}$ & $3,7(1,4)$ & $4,1(1,3)$ & $-2,67 * / 0,27$ \\
\hline $\begin{array}{l}\text { 11. É melhor permanecer com fome quando não há uma opção } \\
\text { alimentar vegetariana ou vegana no local. }\end{array}$ & $4,0(1,3)$ & $4,5(0,9)$ & $-4,64 * / 0,48$ \\
\hline $\begin{array}{l}\text { 12. A companhia de vegetarianos/veganos num restaurante, por } \\
\text { exemplo, não influencia na escolha da minha refeição. }{ }^{1}\end{array}$ & $3,9(1,4)$ & $4,3(1,3)$ & $-2,99 * / 0,30$ \\
\hline $\begin{array}{l}\text { 13. Alguém que se diz vegetariano estrito/vegano, e que faz uso de } \\
\text { mel ou gelatina, pode ter esse título. }{ }^{1}\end{array}$ & $4,4(1,0)$ & $4,6(1,0)$ & $-1,98 * / 0,20$ \\
\hline $\begin{array}{l}\text { 14. Em meio a amigos ou familiares, é melhor comer carne a ser } \\
\text { alvo de piadas ou ofensas por ser vegetariano/vegano. }{ }^{1}\end{array}$ & $5,0(0,3)$ & $5,0(0,3)$ & - \\
\hline $\begin{array}{l}\text { 15. Apesar de vegetariano/vegano, o cheiro de carne não me } \\
\text { incomoda. }{ }^{1}\end{array}$ & $3,5(1,5)$ & $3,9(1,4)$ & $-2,82 * / 0,29$ \\
\hline $\begin{array}{l}\text { 16. Os regimes alimentares vegetarianos são mais saudáveis que os } \\
\text { estilos onívoros (não vegetarianos). }\end{array}$ & $3,9(1,2)$ & $4,3(1,0)$ & $-3,70 * / 0,38$ \\
\hline $\begin{array}{l}\text { 17. Só é vegetariano/vegano quem mudou sua alimentação por } \\
\text { vontade própria, sem ter sido forçado. }\end{array}$ & $3,3(1,5)$ & $3,5(1,5)$ & - \\
\hline $\begin{array}{l}\text { 18. Quem comeu carne por não ter outra opção (ex.: numa } \\
\text { internação hospitalar), não é mais vegetariano/vegano. }\end{array}$ & $1,8(1,1)$ & $2,2(1,3)$ & $-3,38 * / 0,34$ \\
\hline $\begin{array}{l}\text { 19. Quem come carne raramente (ex.: numa ceia de natal) continua } \\
\text { sendo vegetariano/vegano. }{ }^{1}\end{array}$ & $3,9(1,4)$ & $4,5(1,1)$ & $-4,63 * / 0,47$ \\
\hline $\begin{array}{l}\text { 20. Não conseguiria voltar a comer carnes, pois o sabor não me } \\
\text { agrada mais. }\end{array}$ & $3,9(1,3)$ & $4,1(1,3)$ & - \\
\hline
\end{tabular}


Tabela 2.

Médias Geral e por Itens para Cada Grupo, com Valores de Comparação de Médias (Continuação)

\begin{tabular}{|c|c|c|c|}
\hline Itens do questionário & $\begin{array}{c}\mathrm{VNE} \\
(\mathrm{N}=198) \\
M(D P)\end{array}$ & $\begin{array}{c}\mathrm{VE} \\
(\mathrm{N}=200) \\
M(D P)\end{array}$ & $t / d$ \\
\hline $\begin{array}{l}\text { 21. Vegetarianos/veganos podem aceitar carnes oferecidas por } \\
\text { pessoas importantes em suas vidas. }{ }^{1}\end{array}$ & $4,7(0,8)$ & $4,9(0,5)$ & $-2,87 * / 0,30$ \\
\hline $\begin{array}{l}\text { 22. Alguém que não come carne só pode ser chamado de } \\
\text { vegetariano se estiver preocupado com a causa animal. }\end{array}$ & $1,8(1,2)$ & $2,0(1,3)$ & - \\
\hline $\begin{array}{l}\text { 23. Pessoas que não comem carnes por saúde ou estética, ao } \\
\text { pensarem mais nelas, não são verdadeiras vegetarianas. }\end{array}$ & $1,8(1,2)$ & $1,9(1,3)$ & - \\
\hline $\begin{array}{l}\text { 24. Mesmo que um animal estivesse no fim da vida, eu não o } \\
\text { comeria. }\end{array}$ & $4,7(0,9)$ & $4,9(0,6)$ & $-2,13 * / 0,22$ \\
\hline $\begin{array}{l}\text { 25. Vegetarianos e veganos devem se afastar de pessoas que } \\
\text { supervalorizam a carne. }\end{array}$ & $1,3(0,6)$ & $1,5(0,9)$ & $-2,94 * / 0,29$ \\
\hline $\begin{array}{l}\text { 26. Chego a sentir raiva ou tristeza quando vejo carnes, pois } \\
\text { lembro de onde elas vieram. }\end{array}$ & $3,8(1,3)$ & $4,2(1,1)$ & $-3,87 * / 0,40$ \\
\hline $\begin{array}{l}\text { 27. Quem não come carnes por não gostar do sabor é tão } \\
\text { vegetariano quanto quem não come por conta dos animais. }{ }^{1}\end{array}$ & $1,7(1,1)$ & $2,1(1,3)$ & $-3,12 * / 0,26$ \\
\hline 28. As texturas das carnes são desagradáveis para mim. & $3,5(1,4)$ & $3,6(1,5)$ & - \\
\hline $\begin{array}{l}\text { 29. Pessoas que evitam carnes por questões religiosas/espirituais } \\
\text { só serão vegetarianas se pensarem nos animais }\end{array}$ & $1,8(1,1)$ & $2,1(1,3)$ & $-2,50 * / 0,25$ \\
\hline $\begin{array}{l}\text { 30. Vou me sentir culpado se eu comer um alimento com carne } \\
\text { por engano. }\end{array}$ & $3,6(1,6)$ & $3,8(1,5)$ & - \\
\hline $\begin{array}{l}\text { 31. Vegetarianos/veganos que comeram carne deveriam se punir, } \\
\text { para aliviar a culpa e evitar um novo erro. }\end{array}$ & $1,2(0,6)$ & $1,3(0,8)$ & - \\
\hline $\begin{array}{l}\text { 32. Vegetarianos e veganos não precisam evitar locais e eventos } \\
\text { que estão associados a carnes. }{ }^{1}\end{array}$ & $2,0(1,2)$ & $2,6(1,3)$ & $-4,97 * / 0,50$ \\
\hline $\begin{array}{l}\text { 33. Quem não come carnes por influência de outras pessoas só } \\
\text { será vegetariano se entender as razões desse movimento. }\end{array}$ & $2,2(1,3)$ & $2,5(1,5)$ & $-2,15 * / 0,22$ \\
\hline $\begin{array}{l}\text { 34. Vegetarianos/veganos devem lembrar às pessoas, } \\
\text { constantemente, a respeito de como as carnes são obtidas. }\end{array}$ & $2,7(1,3)$ & $3,5(1,3)$ & $-5,95 * / 0,59$ \\
\hline $\begin{array}{l}\text { 35. Não descarto a ideia de, no futuro e a depender das } \\
\text { circunstâncias, voltar a comer carnes. }{ }^{1}\end{array}$ & $4,1(1,3)$ & $4,7(0,7)$ & $-6,01 * / 0,43$ \\
\hline $\begin{array}{l}\text { 36. Eu comeria uma certa carne, sabendo que veio de um animal } \\
\text { que teve vida digna e abate humanizado. }{ }^{1}\end{array}$ & $4,6(0,9)$ & $4,9(0,3)$ & $-5,29 / 0,59 *$ \\
\hline
\end{tabular}

Nota. ${ }^{1}$ Itens invertidos (maiores médias indicam maior discordância).

- Dados ausentes devido à semelhança entre os grupos para esse item.

animal que fundamenta o estilo de vida vegano, no qual se defende que os corpos e vidas dos animais pertencem somente a eles, não estão à serviço dos seres humanos. Essa vertente seria contrária à que propaga a noção de bem-estar animal, no qual os cuidados dispensados aos animais seriam apenas um meio, com a finalidade de disponibilizar um melhor produto para o consumo humano, o chamado bem-estarismo (Ferrigno, 2012).

Além disso, o grupo VE também apresentou maior discordância do que o grupo VNE quanto ao item 27 (Quem não come carnes por não gostar do sabor é tão vegetariano quanto quem não come por 
conta dos animais), porém também com um efeito baixo $(0,26)$. Esses resultados sugerem uma diferença entre os grupos quanto à desvinculação do vegetarianismo da preocupação com os animais, uma vez que a definição de vegetarianismo se baseia nas características alimentares, e não nos motivos que as embasam (Sociedade Vegetariana Brasileira, 2014), sendo o grupo VE mais avesso a essa definição. Porém, as médias similares de discordância entre os grupos quanto ao item 22 (Alguém que não come carne só pode ser chamado de vegetariano se estiver preocupado com a causa animal) sugerem uma interpretação contrária, com o grupo VNE apresentando compreensão similar ao grupo VE quanto a discordar da definição de vegetarianismo apenas com base nas práticas alimentares.

A definição diferente de vegetarianismo entre os grupos, sendo a do grupo VE baseada em princípios além de hábitos alimentares, é evidenciada na maior discordância do grupo VE com o item 8 (Quem não come carnes, e mantém o consumo de ovos ou de laticínios, pode ser chamada de vegetariano) comparado ao grupo VNE, a maior diferença entre os grupos $(d=0,77)$. Além disso, o grupo VE também apresentou mais discordância com o item 2 (Qualquer pessoa que não come carnes pode ser chamada de vegetariana) e com o item 3 (Qualquer pessoa que não come produtos de origem animal pode ser chamada de vegana). Esse dado corrobora Ruby (2012) e Rosenfeld (2018) em termos de discordância, entre o público vegano, quanto a práticas ditas vegetarianas que mantém o consumo de outros itens de origem animal, o que manteria a exploração e o sofrimento de animais. Esse dado corrobora ainda a tendência de considerar vegetarianas somente as práticas alimentares de base unicamente vegetal, enquanto as demais receberiam outras designações como ovolactovegetariana (Ruby, 2012), e ainda de considerar que um estilo vegano, por sua vez, não se restringiria a uma prática alimentar (Ferrigno, 2012)

A definição de vegetariano/vegano reflete-se também na maior concordância do grupo VE com o item 18 (Quem comeu carne por não ter outra opção, como numa internação hospitalar, não é mais vegetariano/vegano), porém o tamanho do efeito é próximo de fraco $(d=0,34)$ além de médias baixas de concordância em ambos os grupos (1,8 para VNE e 2,2 para $\mathrm{VE})$, o que remete à noção do veganismo dentro do possível e do praticável (The Vegan Society, 2014). Em situações onde a capacidade de escolha está comprometida e/ou envolve risco à saúde do indivíduo, por exemplo, a gravidade do consumo de itens de origem animal seria relativizada. Mas somente nessas situações: a alta discordância em ambos os grupos com os itens 19 (Quem come carne raramente, como numa ceia de natal, continua vegetariana/vegana); 21 (Vegetarianos/ veganos podem aceitar carnes oferecidas por pessoas importantes em suas vidas); e 35 (Não descarto a ideia de, no futuro e a depender das circunstâncias, voltar a comer carnes), corroboram essa especificidade na amostra estudada.

Com relação a alimentos específicos, o grupo VE apresentou maior discordância que o grupo VNE com os itens 9 (Um vegetariano que comeu peixe ou fruto do mar errou menos do que outro que comeu carne vermelha ou de ave), 10 (Um vegano que comeu ovo ou laticínio errou menos do que outro que consumiu alguma carne) e 13 (Alguém que se diz vegetariano estrito/vegano, e que faz uso de mel ou gelatina, pode ter esse título), porém com efeito baixo $(0,26 ; 0,27$; e 0,20 , respectivamente). É importante mencionar que os itens 9 e 13 situaram-se entre os maiores níveis de discordância em ambos os grupos. Esses dados podem estar pautados na noção de senciência dos animais, que é a capacidade de experimentar sensações e emoções de forma consciente. Então, o consumo de qualquer item de origem animal seria igualmente reprovável (Rosenfeld, 2018).

Considerando a valorização social em função do comportamento alimentar, o grupo VE apresentou maior concordância que o grupo VNE com o item 5 (Veganos são mais admiráveis que vegetarianos, pois não dependem dos animais para se alimentarem), porém com efeito baixo $(0,27)$. Esse dado alude à hierarquia entre os tipos de práticas vegetarianas, sendo o veganismo o vegetarianismo em seu formato mais puro e, em tese, mais admirável (Jabs et al., 2000). Porém, é importante observar que a média de concordância foi baixa nos dois grupos, sugerindo que veganos não se consideram superiores em relação a vegetarianos.

Valorização e aprovação social também parecem influenciar o comportamento alimentar ao se observar a maior discordância por parte do grupo VE comparado ao VNE com o item 12 (A companhia de vegetarianos/ veganos num restaurante, por exemplo, não influencia na escolha da minha refeição). Esse último dado está alinhado ao estudo de Jabs et al., (2000), que apontam a presença de vegetarianos e veganos como influência sobre a escolha por alimentos, especialmente entre os adeptos do mesmo estilo de vida. Ainda com relação a influenciar outras pessoas, o grupo VE apresentou mais concordância com o item 34 (Vegetarianos/veganos 
devem lembrar às pessoas, constantemente, a respeito de como as carnes são obtidas) do que o grupo VNE, o que demarca um caráter de ativismo maior no movimento vegano, em prol de modos de vida que conciliem a satisfação das necessidades humanas e a libertação animal (Esteves, 2017; The Vegan Society, 2014).

O grupo VE comparado ao grupo VNE apresentou maior concordância quanto ao item 11 (É melhor permanecer com fome quando não há uma opção alimentar vegetariana ou vegana no local), sugerindo serem menos suscetíveis a cederem a consequências imediatas, o que sugere o uso de estratégias de autocontrole. O item 25 (Vegetarianos e veganos devem se afastar de pessoas que supervalorizam a carne) indica uma possível estratégia. Porém, quando se considera a discordância de ambos os grupos em relação a esse item, tal estratégia parece ser pouco usada pelos participantes.

Uma condição de autocontrole envolve a emissão de uma resposta controladora, por parte de um organismo, que afeta a probabilidade de ocorrência de uma outra resposta (Skinner, 1953/2003). Deixar de frequentar certos espaços ou eventos ou interagir menos com algumas pessoas, como onívoros incentivadores do consumo de carne, podem ser exemplos de respostas controladoras, diminuindo as chances de se consumir os produtos cárneos. Porém, a depender das contingências, esses comportamentos podem ser esquivas frente às carnes, tidas como aversivas em suas propriedades físicas e/ou pelos significados que apresentam, os quais aludem às regras.

As regras são descrições verbais que caracterizam contingências, que contemplam os comportamentos de um organismo e as condições em que ocorrem. Os comportamentos são fortalecidos conforme correspondam às regras que os descrevem e sejam seguidos de reforços no meio social (Albuquerque, 2001). Os grupos de redes sociais que reúnem vegetarianos e veganos, por exemplo, ao enfatizarem os preceitos norteadores de tais movimentos e valorizarem atitudes em prol deles, ajudam a fortalecer tais preceitos (regras), as ações descritas (comportamentos alimentares) e a correspondência entre os preceitos e as ações desempenhadas (seguimento de regras).

Discursos frequentes professados por tais grupos, como os que associam comer carne a machucar os animais, podem favorecer uma alimentação isenta de produtos cárneos. Segundo essa ótica, tais grupos emitem variáveis verbais que podem selecionar e manter comportamentos alimentares vegetarianos e respostas autocontroladas associadas por meio de reforços (Reis, Teixeira, \& Paracampo, 2005). Em outras palavras, o suporte social desses grupos, durante a transição e manutenção dos estilos de vida vegetarianos, podem ser um importante componente para a seleção de comportamentos alimentares alinhados ao vegetarianismo e ao veganismo (Skinner, 1981/2007).

Outra comparação realizada entre os grupos se deu em termos de alimentação motivada por valores (MV) (causa animal, ambiental e/ou religiosa/espiritual) e baseada em motivos mistos (MM), que incluíam pelo menos um dos motivos anteriores somados a fatores auto-orientados ou pessoais (saúde, estética e/ou economia financeira), tendo sido incluída nesse grupo também a aversão às propriedades físicas dos itens de origem animal, como sabor. As práticas alimentares norteadas por valores estariam sujeitas a um maior controle de regras, enquanto as de embasamento misto seriam mais sensíveis às contingências imediatas. Fatores unicamente pessoais ou auto-orientados foram tidos como influentes únicos no início da prática vegetariana por apenas 12 participantes e, na manutenção, por nove participantes, e, em função da baixa frequência, não foram discutidos em detalhes.

Para o início da alimentação vegetariana, a concordância média dos participantes que apresentavam MV foi maior em comparação àqueles que apresentaram MM (MV[193]: $M=3,4, D P=0,4 / \mathrm{MM}[191]: M=3,2$, $D P=0,4 / t=3,96, p=0,00, d=0,50)$. Para a manutenção da alimentação vegetariana, também foi verificada uma diferença (MV[117]: $M=3,4, D P=0,4 / \mathrm{MM}[226]$ : $M=3,3, D P=0,4)$ significativa $(t=2,03, p=0,04, d$ $=0,25)$, sugerindo maior restrição entre vegetarianos e veganos com práticas alimentares norteadas unicamente por valores. Esse dado está alinhado aos estudos de Hoffman, Stallings, Bessinger e Brooks (2013) e Rothgerber (2014), que verificaram maior restrição alimentar entre vegetarianos por motivos éticos, em comparação aos que elencavam somente motivos auto-orientados, como saúde pessoal. Vale ressaltar que as noções de restrição e flexibilidade não possuem caráter valorativo. Então, ser mais restritivo no nível alimentar não implicaria na existência de comportamentos inadequados ou prejudiciais à saúde.

Mediante esse cenário, torna-se necessária a implementação de ações de promoção de saúde junto ao público vegetariano, como também aos onívoros, que considerem a multideterminação das escolhas alimentares (Corrin \& Papadopoulos, 2017; Lima, 2018; Rosenfeld \& Burrow, 2017a, 2017b; Ruby et al., 2013). 
Vale ressaltar ainda a importância dos profissionais se atualizarem sobre o tema, uma vez que as dietas vegetarianas são saudáveis e seguras, podendo ser adotadas por diferentes tipos de pessoas, segundo a Associação Americana de Dietética (Melina et al., 2016).

Por outro lado, existem dados que apontam uma relação entre vegetarianismo e transtornos alimentares. Essa associação poderia ser explicada pelo uso de práticas vegetarianas para camuflar problemas de saúde, ou pela restrição implicada na alimentação vegetariana afetar a saúde de pessoas que já estão vulneráveis por motivos outros (Zuromski et al., 2015). Cabe ao profissional de saúde, portanto, diferenciar estilos vegetarianos e veganos saudáveis de práticas que possuem essas denominações, mas que caracterizam adoecimentos físicos e psicológicos.

\section{Considerações Finais}

A maior presença de comportamentos restritivos entre vegetarianos estritos e veganos, como também entre os que associaram tais estilos de vida unicamente a valores, sugere comportamentos com maior sensibilidade a regras entre esses públicos, que teriam mais comportamentos em prol de reforços de longo prazo. Comportamentos sob controle de tais reforços, porém, não estão insensíveis às contingências atuais, o que ajuda a questionar concepções atribuídas aos vegetarianos e veganos como unicamente preocupados com causas distantes e que restringem suas vidas de forma geral. Uma restrição em nível alimentar não necessariamente se generalizaria para outros âmbitos, a exemplo das interações pessoais, o que dependeria de outros fatores.

Como limitações deste estudo, aponta-se a ausência de indicação da região geográfica dos participantes, o que impossibilitou verificar o alcance da pesquisa. É preciso considerar também que esses dados retratam uma amostra específica, de pessoas com nível superior de instrução e ativas em redes sociais. Estudos futuros com outros vegetarianos e veganos, inseridos em outras realidades, poderiam contribuir com a discussão desse tema. Outra limitação se refere à falta de instrumentos psicométricos validados para avaliar o estilo de vida vegetariano. A única escala encontrada, de Rosenfeld e Burrow (2018), foi disponibilizada após o início da coleta de dados do presente estudo, o que inviabilizou o seu uso no presente trabalho.

Quanto a pesquisas futuras, sugere-se que sejam investigadas a qualidade do atendimento em saúde junto aos vegetarianos e veganos, segundo a percepção deles e dos profissionais de saúde, além de estudos avaliando a qualidade de vida dessas pessoas. Outros temas de estudos em Psicologia sobre o vegetarianismo foram trazidos por Rosenfeld (2018), como a influência desse estilo de vida nas relações amorosas e na educação dos filhos, vegetarianismo e vivências religiosas, intersecções entre práticas vegetarianas e noções de masculinidade e feminilidade, dentre outros.

\section{Referências}

Albuquerque, L. C. (2001). Definições de regras. Em H. J. Guilhardi, M. B. B. P. Madi, P. P. Queiroz \& M. C. Scoz (Eds.). Sobre comportamento e cognição: expondo a variabilidade (pp. 259-274). Santo André: ARBytes.

Aleksandrowicz, L., Green, R., Joy, E. J. M., Smith, P., \& Haines, A. (2016). The impacts of dietary change on greenhouse gas emissions, land use, water use, and health: a systematic review. PLoS One, 11(11):e0165797. doi:10.1371/journal. pone. 0165797

Allès, B., Baudry, J., Mejean, C., Touvier, M., Peneau, S., Hercberg, S., \& Kesse-Guyot, E. (2017). Comparison of sociodemographic and nutritional characteristics between self-reported vegetarians, vegans, and meat-eaters from the Nutrinet-Sante study. Nutrients, 9, 1023. doi: 10.3390/nu9091023

Arora, A. S., Bradford, S., Arora, A., \& Gavino, R. (2017). Promoting vegetarianism through moralization and knowledge calibration, Journal of Promotion Management, 23(6), 889-912, doi: 10.1080/10496491.2017.1323263

Beardsworth, A. D., \& Keil, E. T. (1992). The vegetarian option: Varieties, conversions, motives and careers. Sociological Review, 40(2): 253-293. doi: 10.1111/j. 1467-954X.1992.tb00889.x

Brasil (2016). Resolução $n^{\circ}$ 510, de 7 de abril de 2016, do Conselho Nacional de Saúde. Ministério da Saúde. Diário Oficial da União.

Corrin, T., \& Papadopoulos, A. (2017). Understanding the attitudes and perceptions of vegetarian and plant-based diets to shape future health promotion programs. Appetite, 109, 40-47. doi: 10.1016/j. appet.2016.11.018

Esteves, L. O. B. (2017). "Eles querem nos converter": Representações sociais sobre a minoria ativa vegan (Dissertação 
de mestrado). Instituto de Psicologia, Universidade de Brasília, Brasilia, Brasil.

Ferrigno, M. V. (2012). Veganismo e libertação animal: Um estudo etnográfico (Dissertação de mestrado). Instituto de Filosofia e Ciências Humanas, Universidade Estadual de Campinas, Campinas, Brasil.

Forestell, C. A., \& Nezlek, J. B. (2018). Vegetarianism, depression, and the five factor model of personality. Ecology of Food and Nutrition, 57, 246-59. doi: 10.1080/03670244.2018.1455675

Hamilton, M. (2006). Eating death. Vegetarians, meat, and violence. Food Culture and Society, 9, 155-177. doi: 10.2752/155280106778606053.

Hoffman, S. R., Stallings, S. F., Bessinger, R. C., \& Brooks, G. T. (2013). Differences between health and ethical vegetarians. Strength of conviction, nutrition knowledge, dietary restriction, and duration of adherence. Appetite, 65, 139-144. doi: 10.1016/j. appet.2013.02.009

Ibope (2018). 14\% da população se declara vegetariana. Recuperado de http://www.ibopeinteligencia.com/ noticias-e-pesquisas/14-da-populacao-se-declara-vegetariana/

Jabs, J., Devine, C. M., \& Sobal, J. (1998). Maintaining vegetarian diets: Personal factors, social networks and environmental resources. Journal of the Canadian Dietetic Association, 59(4), 183-189.

Jabs J., Sobal, J., \& Devine, C. M. (2000). Managing vegetarianism: Identities, norms, and interactions. Ecology of Food and Nutrition, 39, 375-394. doi: 10.1080/03670244.2000.9991625

Lima, P. P. F. (2018). A construção social da alimentação: $O$ vegetarianismo e o veganismo na perspectiva da psicologia histórico cultural (Tese de doutorado). Instituto de Psicologia, Universidade Federal da Bahia, Salvador, Brasil.

Melina, V., Craig, W., \& Levin, S. (2016). Position of the academy of nutrition and dietetics: Vegetarian diets. Journal of the Academy of Nutrition and Dietetics, 116, 1970-1980. doi: 10.1016/j. jand.2016.09.025

Pfeiler, T. M., \& Egloff, B. (2018). Examining the "Veggie" personality: Results from a representative German sample. Appetite, 120, 246-255. doi: 10.1016/j.appet.2017.09.005.
Reis, A. A., Teixeira, E. R., \& Paracampo, C. C. P. (2005). Autorregras como variáveis facilitadoras na emissão de comportamentos autocontrolados: $\mathrm{O}$ exemplo do comportamento alimentar. Interação em Psicologia, 9(1), 57-64. doi: 10.5380/psi.v9i1.3286

Rosenfeld, D. L. (2018). The psychology of vegetarianism: Recent advances and future directions. Appetite, 131, 125-138. doi: 10.1016/j.appet.2018.09.011

Rosenfeld, D. L., \& Burrow, A. L. (2017a). The unified model of vegetarian identity: A conceptual framework for understanding plant-based food choices. Appetite, 112, 78-95. doi: 10.1016/j. appet.2017.01.017

Rosenfeld, D. L., \& Burrow, A. L. (2017b). Vegetarian on purpose: Understanding the motivations of plant-based dieters. Appetite, 116, 456-463. doi: 10.1016/j.appet.2017.05.039

Rosenfeld, D. L., \& Burrow, A. L. (2018). Development and validation of the Dietarian Identity Questionnaire: Assessing self-perceptions of animal-product consumption. Appetite, 127, 182-194. doi:10.1016/j.appet.2018.05.003

Rothgerber, H. (2014). A comparison of attitudes toward meat and animals among strict and semivegetarians, Appetite, 72, 98-105. doi: 10.1016/j. appet.2013.10.002

Ruby, M. B. (2012). Vegetarianism. A blossoming field of study. Appetite, 58, 141-150. doi: 10.1016/j. appet.2011.09.019

Ruby, M. B., Heine, S. J., Kamble, S., Cheng, T. K, \& Waddar, M. (2013). Compassion and contamination. Cultural differences in vegetarianism. Appetite, 71, 340-348. doi: 10.1016/j.appet.2013.09.004

Skinner, B. F. (2003). “Autocontrole”. Em B. F. Skinner (Ed.). Ciência e comportamento humano. (11 ed, pp. 249-264). São Paulo: Martins Fontes. (Trabalho original publicado em 1953).

Skinner, B. F. (2007). Seleção por consequências. Revista Brasileira de Terapia Comportamental e Cognitiva, 9, 129-137. (Trabalho original publicado em 1981). doi: 10.31505/rbtcc.v9i1.150

Sociedade Vegetariana Brasileira (2014). Estatuto da Sociedade Vegetariana Brasileira. Recuperado de http:/ / www.svb.org.br/svb/estatuto 
The Vegan Society (2014). Ripened by human determination: Seventy years of the vegan society. Recuperado de https://www.vegansociety.com/sites/default/files/ uploads/Ripened $\% 20$ by $\% 20$ human $\% 20$ determination.pdf

Wolk, A. (2017). Potential health hazards of eating red meat. Journal of Internal Medicine, 281, 106-122. doi: 10.1111/joim.12543
Zuromski, K. L., Witte, T. K., Smith, A. R., Goodwin, N., Bodell, L. P., Bartlett, M., \& Siegfried, N. (2015). Increased prevalence of vegetarianism among women with eating pathology. Eating Behaviors, 19, 24-27. doi: 10.1016/j.eatbeh.2015.06.017

Recebido em: 21/11/2018

Reformulado em: 03/06/2019

Aprovado em: 09/09/2019

Sobre os autores:

Rodrigo Clementino de Carvalho é psicólogo pela Universidade Federal do Vale do São Francisco (2018). Atualmente é psicólogo clínico e atua, principalmente, nas áreas de Comportamento Alimentar e Imagem Corporal.

ORCID: https://orcid.org/0000-0002-5873-036X

E-mail: rodrigoclementinoc@gmail.com

Júnnia Maria Moreira é psicóloga pela Universidade Federal de São João del-Rei (2003), mestre em Psicologia pela Universidade de Brasília (2007) e doutora pela Universidade de Brasília (2015). Atualmente é professora adjunta/DE da Universidade Federal do Vale do São Francisco, campus Petrolina/PE, atuando nas seguintes áreas: Economia Comportamental, Impulsividade e Autocontrole, Análise do Comportamento Aplicada, Habilidades Sociais e Controle Instrucional.

ORCID: https://orcid.org/0000-0003-1167-111X

E-mail: junnia.moreira@gmail.com

Contato com os autores:

Colegiado de Psicologia, Campus Sede da UNIVASF

Av. José de Sá Maniçoba, S/N, Centro

Petrolina-PE, Brasil

CEP: 5604-917 